Check for updates

Cite this: Mater. Adv., 2021, 2, 7701

Received 9th September 2021, Accepted 23rd September 2021

DOI: $10.1039 / \mathrm{d} 1 \mathrm{ma} 00830 \mathrm{~g}$

rsc.li/materials-advances

\title{
A novel aggregation-induced emission poly(phosphoramidate): fluorescence properties and visual detection for $\mathrm{Cu}^{2+} \uparrow$
}

\author{
Liulong Guo, (D) Hongxia Yan, (D) * Lianlian Wang, Pengfei Yang, Lirong Yan and \\ Liulong Guo,
Yan Zhao*
}

\begin{abstract}
Non-aromatic aggregation-induced emission (AIE) polymers have become a hot spot in the materials fields due to their low toxicity and excellent environmental friendliness. However, this kind of polymer usually suffers from insufficient fluorescence properties and limited varieties. Herein, we have synthesized a novel kind of non-aromatic AIE polymer-poly(phosphoramidate) (PPA) for the first time in consideration of the electron-rich properties of $\mathrm{P}$ atoms and excellent biocompatibility of phosphate esters. The PPA is synthesized through a green and facile one-pot ester ammonolysis of triethyl phosphate with ethylenediamine (P1-P3) or 1,6-diaminohexane (P4). The synthesized PPA shows typical AIE characteristics and can emit bright blue fluorescence under UV light. We found that the molecular weight, terminal groups and chain length all have remarkable influence on its fluorescence properties. Transmission electron microscope (TEM) images and density functional theory (DFT) calculations reveal that the fluorescence of PPA can be attributed to the formation of a luminogen cluster and the electronic delocalization caused by through-space conjugation. Notably, PPA is sensitive to $\mathrm{Cu}^{2+}$ and it can change the colour of $\mathrm{CuCl}_{2}$ solution from colourless to purple. Thus, this novel PPA not only enriches the species of non-aromatic AIE polymers, but also provides a convenient visual detection

agent for $\mathrm{Cu}^{2+}$
\end{abstract}

\section{Introduction}

Compared with conventional aggregation-induced emission (AIE) luminogens ${ }^{1,2}$ non-aromatic AIE polymers ${ }^{3,4}$ are emerging in biological imaging, metal ion detection and other fields ${ }^{1,4,5}$ due to their low toxicity and excellent environmental friendliness. However, the species of non-aromatic AIE polymers are mainly limited to $\mathrm{N}$-containing polymers such as poly(amino ester) $\mathrm{s},{ }^{6-8}$ poly(amidoamine)s, ${ }^{9}$ and polyethylenimines. ${ }^{10}$ Recently, our group synthesized a series of Si-containing non-aromatic AIE polymershyperbranched polysiloxane (HBPSi) ${ }^{11-14}$-and found that they can emit bright fluorescence and show a strong AIE effect. Similarly, in consideration of the electron-rich properties of $\mathrm{P}$ atoms and excellent biocompatibility of P-containing polymers, the poly(phosphoramidate) (PPA) can be expected as a novel non-aromatic AIE polymer; however, there has been insufficient study of this kind of polymer until now.

Key Laboratory of Polymer Science and Technology of Shaanxi Province, School of Chemistry and Chemical Engineering, Northwestern Polytechnical University, Xi'an 710072, China.E-mail: hongxiayan@nwpu.edu.cn, zhaoyan@nwpu.edu.cn

$\dagger$ Electronic supplementary information (ESI) available. See DOI: 10.1039/d1ma00830g
Phosphorous polymers mainly include polyphosphoesters, polyphosphoric acids and poly(phosphonamidites) (PPA). Among these polymers, PPA shows good degradability, low toxicity and good designability and has been widely used as a flame retardant. ${ }^{15-18}$ Up to now, PPAs have mostly been synthesized via polycondensation by phosphorus oxychloride and diamine,${ }^{19-22}$ accompanied by hydrogen chloride as a byproduct. However, the reactant phosphorus oxychloride is dangerous due to its strong corrosiveness and the removal of hydrogen chloride complicates the synthesis process. Besides, the fluorescence properties of PPA are seldom studied. Such drawbacks greatly limit the applications of PPA. Thus, the development of an innovative strategy to prepare PPA and a systematic study of its fluorescence property is highly desirable.

Herein, we creatively develop a green and facile one-pot strategy to synthesize PPA through an ester ammonolysis reaction for the first time. By using triethyl phosphate (TEP), ethylenediamine (EDM) and 1,6-diaminohexane (DAH) as reactants, the synthesis process is simple, safe and without complicated separation procedures. Most importantly, the synthesized PPA can emit bright blue fluorescence under UV light and show an AIE effect. We systematically investigated the influence of molecular weight, terminal groups and chain 
length on the fluorescence property of PPA. Transmission electron microscopy (TEM) and density functional theory (DFT) were also applied to explore the fluorescence mechanism of PPA. Moreover, the synthesized PPA could change the colour of $\mathrm{CuCl}_{2}$ solution from colourless to purple. Thus, it can be used as an on-site analysing chromogenic reagent for $\mathrm{Cu}^{2+}$ instead of time-consuming and expensive detection methods, such as electroanalytical chemistry, ${ }^{23}$ atomic absorption spectrometry $^{24}$ or inductively coupled plasma mass spectrometry. ${ }^{25}$ This work provides a green, facile and safe approach to synthesize PPA, and develop a new kind of P-containing non-aromatic AIE polymer. The synthesized PPA is sensitive to $\mathrm{Cu}^{2+}$ and can be used as a chromogenic reagent for the on-site visual detection of $\mathrm{Cu}^{2+}$.

\section{Experimental}

\subsection{Materials}

Triethyl phosphate (TEP, AR) was purchased from Shanghai Macklin Biochemical Co., Ltd. Ethylenediamine (EDM, AR) and 1,6-diaminohexane (DAH, AR) were obtained from Tianjin Tianli Chemical Reagents Co., Ltd. Absolute ethanol (AR) was supplied by Guangdong Guanghua Sci-Tech Co., Ltd. Sodium hydroxide was purchased from Tianjin Jinbei Fine Chemical Co., Ltd. Regenerated cellulose (RC) dialysis membranes with a molecular weight cut off (MWOC) of 1000 were purchased from Xi'an Yobios Biotechnology Co., Ltd. The RC dialysis membranes were cut off and boiled in distilled water for 15 minutes. Other materials were used without further purification.

\subsection{Methods}

Nuclear magnetic resonance spectra $\left({ }^{1} \mathrm{H},{ }^{13} \mathrm{C},{ }^{31} \mathrm{P}\right.$ NMR $)$ were detected on a Bruker Avance $400 \mathrm{MHz}$ superconducting Fourier digital NMR spectrometer using dimethyl sulfoxide (DMSO-d6) as the solvent. Fourier Transform infrared spectroscopy (FT-IR) was recorded on a Bruker infrared spectrometer with a wavenumber ranging from 4000 to $400 \mathrm{~cm}^{-1}$. Ultraviolet-visible (UV-vis) absorption spectroscopy was measured using a HITACHI U-3900H UV-visible absorption spectrometer. Fluorescent excitation/emission spectra of PPA solution were measured on a F-30 fluorescence spectrophotometer with a scanning speed of $240 \mathrm{~nm} \mathrm{~min}^{-1}$. Fluorescent excitation/emission spectra, fluorescence lifetime and absolute quantum yield (QY) of the pure PPA were measured on a UK Edinburgh FLS980 fullfeatured steady-state/transient fluorescence spectrometer at room temperature. A $340 \mathrm{~W}$ xenon lamp was used as the light source. Gel permeation chromatography (GPC) was used to measure the molecular weight and distribution of the PPA, with $0.1 \mathrm{M} \mathrm{NaNO}$ aqueous solution as eluent with a flow rate of $1 \mathrm{~mL} \mathrm{~min}^{-1}$ at room temperature. The density functional theory (DFT) method at the B3LYP/6-31 G (d) level was used to calculate the energy level of the PPA. Transmission electron microscopy (TEM) was applied to observe the morphology of PPA clusters on a FEI Tecnai G2 F20 microscope. The measurement was performed at $200 \mathrm{kV}$ and room temperature. The cell biocompatibility of PPA was assessed by CCK-8 assays.

\subsection{Synthesis of PPA}

The specific synthesis method is as follows. First, $27.32 \mathrm{~g}$ $(0.15 \mathrm{~mol})$ of TEP and $14.42 \mathrm{~g}(0.24 \mathrm{~mol})$ of EDM (the ratio of $-\mathrm{OCH}_{2} \mathrm{CH}_{3}$ to $-\mathrm{NH}_{2}$ is $3: 3.2$ ) were added to a three-necked $100 \mathrm{~mL}$ flask equipped with a nitrogen gas inlet, a condenser and a mechanical stirrer. The mixture was stirred at an appropriate rate. Subsequently, the system was slowly heated to $85{ }^{\circ} \mathrm{C}$ to reach the reaction temperature. This temperature was kept for 8 hours. Then, the temperature was increased by $10{ }^{\circ} \mathrm{C}$, lasting for 4 hours. Finally, the light-yellow viscous liquid was collected into the vial as the product cooled.

The product was dissolved in an appropriate amount of ethanol and loaded into the RC dialysis membranes. After 24 hours of dialysis, steaming was carried out to remove the solvent. After the steaming, the sample was placed in a vacuum oven overnight. The product PPA (named P1) was then obtained.

${ }^{1} \mathrm{H}$ NMR (400 Hz, DMSO-d6): $\delta 3.69$ (p,16H), $3.44(\mathrm{q}, 1 \mathrm{H}), 2.77$ (m, 14H), $2.63(\mathrm{dq}, 1 \mathrm{H}), 1.11(\mathrm{~m}, 30 \mathrm{H})$.

P2 was synthesized using $27.32 \mathrm{~g}(0.15 \mathrm{~mol})$ of TEP and $12.60 \mathrm{~g}(0.21 \mathrm{~mol})$ of EDM (the ratio of $-\mathrm{OCH}_{2} \mathrm{CH}_{3}$ to $-\mathrm{NH}_{2}$ is $3: 2.8$ ) in a similar synthesis process to $\mathrm{P} 1$.

P3 was synthesized using $27.32 \mathrm{~g}(0.15 \mathrm{~mol})$ of TEP and $16.20 \mathrm{~g}(0.27 \mathrm{~mol})$ of EDM (the ratio of $-\mathrm{OCH}_{2} \mathrm{CH}_{3}$ to $-\mathrm{NH}_{2}$ is $3: 3.6)$ based on the synthesis process for $\mathrm{P} 1$.

P4 was synthesized using $27.32 \mathrm{~g}(0.15 \mathrm{~mol})$ of TEP and $31.38 \mathrm{~g}(0.27 \mathrm{~mol})$ of 1,6-diaminohexane (the ratio of $-\mathrm{OCH}_{2} \mathrm{CH}_{3}$ to $-\mathrm{NH}_{2}$ is $\left.3: 3.6\right)$ based on the synthesis process for $\mathrm{P} 1$. The reaction temperature was $120{ }^{\circ} \mathrm{C}$.

${ }^{1} \mathrm{H}$ NMR (400Hz, DMSO-d6): $\delta 3.68(\mathrm{p}, 1 \mathrm{H}), \delta 2.57(\mathrm{~m}, 1 \mathrm{H}), \delta$ $1.50(\mathrm{tq}, 1 \mathrm{H}), \delta 1.28(\mathrm{q}, 1 \mathrm{H}), \delta 1.08(\mathrm{dt}, 2 \mathrm{H})$.

The synthesis route and specific synthesis data of PPA are shown in Scheme 1 and Table S1 (ESI $\dagger$ ). The schematic structures of P1-P4 are shown in Fig. S1 (ESI $\dagger$ ). Moreover, the possible reaction mechanism is exhibited in Scheme $\mathrm{S} 1$ ( $\mathrm{ESI} \dagger$ ). It is notable that the reactivity of EDM is higher than that of DAH, so we selected P1 as the main research object.

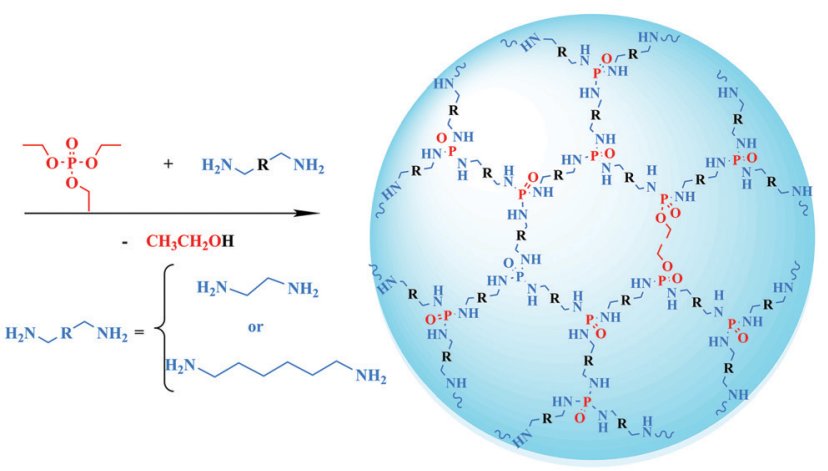

Scheme 1 The synthetic route of PPA. 


\section{Results and discussion}

Distinct from the current method using phosphorus oxychloride for the synthesis of PPA, in this work, P1-P4 were obtained using TEP and EDM or DAH via one-pot ester aminolysis. In addition, no solvent was applied during the process and the byproduct ethanol can be easily removed by distillation. Thus, the proposed synthesis method is simple, safe and has no complicated purification steps.

\section{$3.1 \quad$ FT-IR study}

FT-IR was carried out to determine the chemical structure of the obtained PPA, and the spectra of TEP, EDM, DAH, P1 and $\mathrm{P} 4$ are shown in Fig. 1. In Fig. 1A, the band at about $3390 \mathrm{~cm}^{-1}$ is related to the telescopic vibration of the amino groups $\left(-\mathrm{NH}_{2}\right)$. The telescopic vibration of $-\mathrm{CH}_{2}-$ is observed at around $2965 \mathrm{~cm}^{-1}$. The absorption peak of the bending vibration of $\mathrm{N}-\mathrm{H}$ appears at about $1377 \mathrm{~cm}^{-1}$. The bands at $1215 \mathrm{~cm}^{-1}$ and $1058 \mathrm{~cm}^{-1}$ belong to $\mathrm{P}=\mathrm{O}$ and $\mathrm{C}-\mathrm{N}$, respectively. In Fig. $1 \mathrm{~B}$, the adsorption peak at around $3325 \mathrm{~cm}^{-1}$ is classified as the telescopic vibration of the amino groups. The adsorption peaks of $\mathrm{P}=\mathrm{O}$ and $\mathrm{C}-\mathrm{N}$ appear at about $1225 \mathrm{~cm}^{-1}$ and $1051 \mathrm{~cm}^{-1}$, respectively, while the bands at $1035 \mathrm{~cm}^{-1}(\mathrm{C}-\mathrm{O})$ and $929 \mathrm{~cm}^{-1}$ (P-O) disappear. The obtained FT-IR spectra offer obvious evidence for the synthesis of the designed PPA.

\subsection{NMR study and degree of branching of PPA}

To further confirm the structure of the synthesized PPA, we obtained ${ }^{1} \mathrm{H},{ }^{13} \mathrm{C}$ and ${ }^{31} \mathrm{P}$ NMR spectra of TEP, EDM, DAH, P1-P4 (Fig. 2 and Fig. S2-S4, ESI $\dagger$ ). In the spectrum of TEP in Fig. 2A, the proton peaks marked by 1 and 2 are associated with $-\mathrm{OCH}_{2} \mathrm{CH}_{3}$ and $-\mathrm{OCH}_{2} \mathrm{CH}_{3}$, respectively. While the peaks at about 2.50 and 3.30 are consistent with DMSO-d6 and water. In the EDM spectra (Fig. 2A), the proton peaks marked by 1 and 2 are associated with $-\mathrm{NH}_{2}$ and $-\mathrm{CH}_{2}-$. In the ${ }^{1} \mathrm{H}$ NMR spectra of $\mathrm{P} 1$, the signal marked by 4 at $\delta=1.11 \mathrm{ppm}$ belongs to $-\mathrm{NH}-$. The signal marked by 2 at $\delta=2.63 \mathrm{ppm}$ is related to $-\mathrm{CH}_{2}-\mathrm{NH}_{2}$. The signal of $-\mathrm{CH}_{2}-\mathrm{NH}-$ appears at $\delta=2.77 \mathrm{ppm}$ is marked by 3 . The peak marked by 1 at $3.69 \mathrm{ppm}$ comes from $-\mathrm{NH}_{2}$. The detailed and analytical ${ }^{1} \mathrm{H}$ NMR spectra of $\mathrm{P} 1$ are displayed in Fig. $2 \mathrm{~B}$. In the DAH spectra (Fig. 2C), the proton peaks marked by 2, 3 and 4 are associated with $\mathrm{H}_{2} \mathrm{~N}-\mathrm{CH}_{2}-\mathrm{CH}_{2}-\mathrm{CH}_{2}-\mathrm{CH}_{2}-\mathrm{CH}_{2}-\mathrm{CH}_{2}-\mathrm{NH}_{2}$ (2.49$2.52 \mathrm{ppm}), \mathrm{H}_{2} \mathrm{~N}-\mathrm{CH}_{2}-\mathrm{CH}_{2}-\mathrm{CH}_{2}-\mathrm{CH}_{2}-\mathrm{CH}_{2}-\mathrm{CH}_{2}-\mathrm{NH}_{2}$ 1.28 ppm), and $\mathrm{H}_{2} \mathrm{~N}-\mathrm{CH}_{2}-\mathrm{CH}_{2}-\mathrm{CH}_{2}-\mathrm{CH}_{2}-\mathrm{CH}_{2}-\mathrm{CH}_{2}-\mathrm{NH}_{2}$
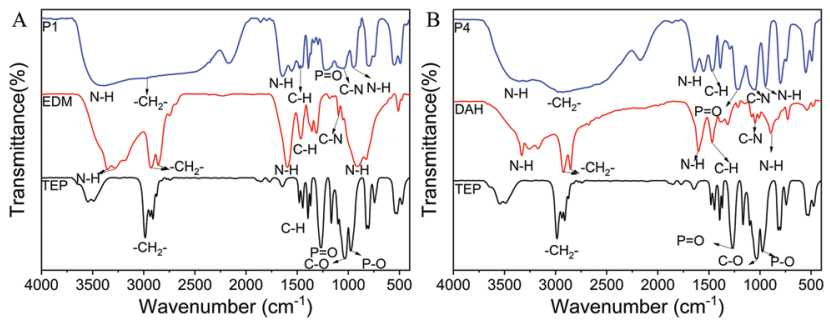

Fig. 1 (A) FT-IR spectra of TEP, EDM and P1; (B) FT-IR spectra of TEP, DAH and P4.
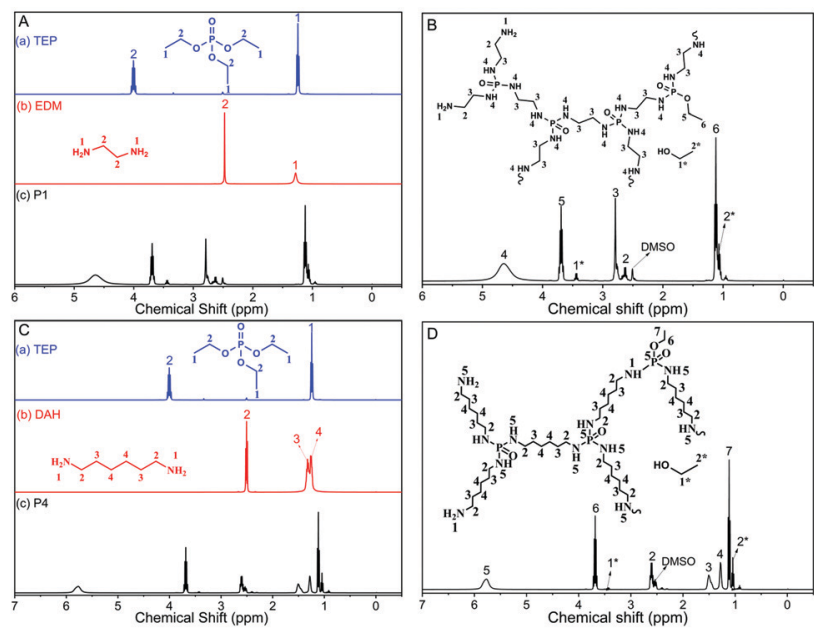

Fig. 2 (A) ${ }^{1} \mathrm{H}$ NMR spectra of TEP, EDM and $\mathrm{P} 1 ;$ (B) ${ }^{1} \mathrm{H}$ NMR spectra of $\mathrm{P} 1$; (C) ${ }^{1} \mathrm{H}$ NMR spectra of TEP, DAH and P4; (D) ${ }^{1} \mathrm{H}$ NMR spectra of P4.

(1.32-1.37 ppm) respectively. In Fig. 2D, it can be seen that the proton peak associated with $\mathrm{CH}_{2}-\mathrm{NH}_{2}$ arises at 3.65-3.72 ppm, while the proton peaks at 2.51-2.61 ppm are associated with $\mathrm{CH}_{2}-\mathrm{NH}-\mathrm{P}$ and $\mathrm{CH}_{2}-\mathrm{NH}_{2}$. The proton peaks associated with $\mathrm{CH}_{2}-\mathrm{CH}_{2}-\mathrm{NH}-\mathrm{P}$ (or $\mathrm{CH}_{2}-\mathrm{CH}_{2}-\mathrm{NH}_{2}$ ), $\mathrm{CH}_{2}-\mathrm{CH}_{2}-$ $\mathrm{CH}_{2}-\mathrm{NH}-\mathrm{P}$ (or $\mathrm{CH}_{2}-\mathrm{CH}_{2}-\mathrm{CH}_{2}-\mathrm{NH}_{2}$ ) and $\mathrm{CH}_{2}-\mathrm{NH}-\mathrm{P}$ appear at 1.44-1.53 ppm, 1.26-1.30 ppm and 1.03-1.13 ppm, respectively. The ${ }^{1} \mathrm{H}$ NMR analysis of P2-P3 as well as the ${ }^{13} \mathrm{C}$ and ${ }^{31} \mathrm{P}$ NMR analysis of reactants and P1-P4 are shown in Fig. S2-S4 (ESI $\dagger$ ). Furthermore, the degree of branching (DB) is an important parameter of hyperbranched polymers. We calculated the DB of $\mathrm{P} 1-\mathrm{P} 4$ according to the ${ }^{1} \mathrm{H}$ NMR data, and the results are shown in Table $\mathrm{S} 2$ (ESI $\dagger$ ).

The NMR spectra and DB value further confirm that PPA (P1-P4) have been successfully synthesized through the proposed one-pot ester aminolysis method.

\subsection{GPC Study}

The GPC data including the weight average molecular weight $\left(M_{\mathrm{w}}\right)$, number average molecular weight $\left(M_{\mathrm{n}}\right)$ and $Ð\left(M_{\mathrm{w}} / M_{\mathrm{n}}\right)$ of P1-P4 are shown in Fig. S5 (ESI $\dagger$ ). For P1, the $M_{\mathrm{w}}, M_{\mathrm{n}}$ and $\mathrm{D}$ are 16500,10600 and 1.56, respectively, which verified that the synthesized P1 is a polymer with a high molecular weight.

The FT-IR spectra, NMR spectra and GPC results sufficiently prove that PPA has been synthesized successfully.

\subsection{Optical properties}

The obtained PPA is a light-yellow viscous liquid in sunlight, but it can emit intense fluorescence under a $365 \mathrm{~nm}$ UV lamp, hence, we systematically studied the optical properties of PPA for the first time. The results showed that the fluorescence of PPA is closely related to the concentration of PPA: that is, a dilute solution of PPA shows weak fluorescence but the pure state of PPA can emit strong blue fluorescence, which indicates that PPA is a novel AIE polymer.

Subsequently, we employ ethanol and tetrahydrofuran (THF) as a good solvent and a poor solvent to further verify the AIE 

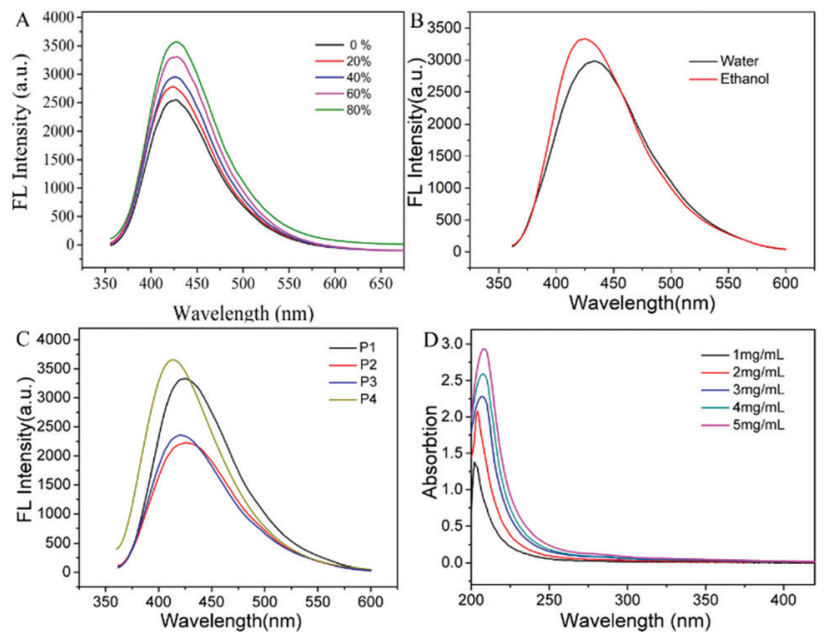

Fig. 3 (A) Fluorescence spectra of $\mathrm{P} 1$ at $20 \mathrm{mg} \mathrm{mL}^{-1}$ in THF/ethanol mixtures with different fractions of THF $\left(\lambda_{\mathrm{ex}}=345 \mathrm{~nm}\right)$; (B) fluorescence spectra of P1 in different solvents $\left(\lambda_{\mathrm{ex}}=345 \mathrm{~nm}\right)$; (C) fluorescence spectra of P1, P2, P3 and P4 ( $\left.\lambda_{\text {ex }}=345 \mathrm{~nm}\right)$; (D) UV-vis absorption spectra of $\mathrm{P} 1$ solution at various concentrations.

character of PPA. In ethanol solution, P1 emits relatively weak blue fluorescence with a wavelength of $415 \mathrm{~nm}$. With an increase in THF fraction $\left(f_{\mathrm{T}}\right)$, the emission intensity gradually increases. The fluorescence intensity increased by $41 \%$ in the ethanol/THF mixture $\left(f_{\mathrm{T}}=80 \%\right)$ compared to that in pure ethanol solution. It should be noted that, according to classical AIE theory, the ethanol solution of PPA should show barely any fluorescence. But in this experiment, due to the physicochemical properties of the polymer, it will form a partial aggregation driven by hydrogen bonds even in good solvents (Fig. S6, ESI $\dagger$ ), resulting in relatively strong fluorescence. With the addition of a poor solvent, the degree of aggregation of the polymer will further be enhanced, and thus its fluorescence intensity will also increase to a certain extent (Fig. 3A). Such a phenomenon is consistent with the AIE effect.

To our surprise, PPA is soluble both in water and in ethanol. This property is undoubtedly beneficial to the application of PPA. Then we investigated the fluorescence properties of P1 solution in water and in ethanol, respectively. As can be seen in Fig. 3B, P1 can emit strong fluorescence both in water and ethanol, but its fluorescence intensity is stronger in ethanol. This could be caused by the different polarities of water and ethanol. It is well known that the polarity of water is stronger than that of amino groups, which makes it more soluble in water. So P1 tends to form tighter aggregates in ethanol than in water. The formation of polymer aggregates reduces the nonradiative loss of energy, so the fluorescence intensity of P1 in ethanol is stronger than that in water. The strong fluorescence intensity in aqueous solution and good biocompatibility of PPA indicate great potential in chemical sensing and bio-imaging.

In order to explore the relationship between the fluorescence properties of PPA and its molecular structure, four kinds of PPA were prepared. P1-P3 are synthesized with the TEP to EDM ratios at $1: 1.6,1: 1.4,1: 1.8$, respectively, and $\mathrm{P} 4$ is prepared with the TEP to DAH ratio at $1: 1.6$. The photoluminescence properties of $\mathrm{P} 1-\mathrm{P} 4$ in ethanol solution are shown in Fig. 3C. As can be seen in Fig. 3C, P4 shows the strongest fluorescence intensity and P2 with ethoxy termination shows the lowest. By comparing the fluorescence intensity of ethoxy terminated P2 and primary amine terminated P3, we can draw the conclusion that a primary amine group is not the key factor for the fluorescence of the PPA, but it can increase the fluorescence intensity. Apart from the functional group, molecular weight is another factor that can influence the fluorescence intensity of PPA. P1 has a higher molecular weight than P3 (data shown in Fig. S5, ESI $\dagger$ ) and exhibits stronger fluorescence intensity than P3, indicating that the fluorescence intensity of PPA is positively correlated with its molecular weight. Compared with P1, P4 has a smaller molecular weight and a longer chain. As shown in Fig. 3C, the fluorescence intensity of P4 is stronger than that of $\mathrm{P} 1$, indicating that the chain length plays a more important role than molecular weight in affecting the fluorescence of PPA.

The UV absorption spectra of P1 in ethanol solution were acquired at diverse concentrations. The detailed spectra are shown in Fig. 3D. The UV absorption band of PPA is mainly concentrated around $205 \mathrm{~nm}$, which is caused by the $n-\pi^{*}$ electronic transition of $\mathrm{C}-\mathrm{NR}_{2}$ and $\mathrm{O}=\mathrm{P}-\mathrm{NH}$. Along with the increase in the PPA concentration, UV absorption intensity is gradually enhanced. The enhancement is attributed to the clusterization of $-\mathrm{NH}_{2}, \mathrm{P}-\mathrm{O}$ and $\mathrm{P}=\mathrm{O}$ with the growing concentration of PPA. We speculate that such a phenomenon is caused by the formation of an "electronic delocalization system" 26 as the concentration increases, which decreases the energy gap between the HOMO and LUMO orbitals and makes it easier to trigger an electronic transition, resulting in an enhancement in the absorption intensity. The formed electronic delocalization system also leads to a red-shift of the absorption wavelength.

Subsequently, we investigated the fluorescence properties of P1 at different concentrations. As the concentration of P1 increases, the fluorescence intensity gradually increases, showing a significant AIE property. The specific fluorescence excitation/emission spectra of P1 at different concentrations are shown in Fig. 4A. Apparently, both the excitation and emission intensity increase with the increase in P1 concentration. In Fig. 4A, the excitation bands of P1 appear at around 262 and $345 \mathrm{~nm}$, while the emission band appears at about $425 \mathrm{~nm}$. The excitation band at around $262 \mathrm{~nm}$ red-shifts to $272 \mathrm{~nm}$ as the concentration increases, and the shapes of the excitation band at $345 \mathrm{~nm}$ and the emission band at $425 \mathrm{~nm}$ are symmetric as mirror images. It is obvious that the peak intensity at around $262 \mathrm{~nm}$ increases more slowly than that at $345 \mathrm{~nm}$ as the concentration increases. It is believed that the band at $262 \mathrm{~nm}$ belongs to the molecular skeleton of $\mathrm{P} 1$, while the band at about $345 \mathrm{~nm}$ is attributed to the formed electronic delocalization system. As the concentration increases, the fluorescence is mainly caused by the electronic delocalization system rather than the molecular skeleton. In contrast to P1, there is only one band in the excitation spectra of P4 (Fig. 4B). Such results indicate that the molecular skeleton of $\mathrm{P} 4$ can hardly emit 

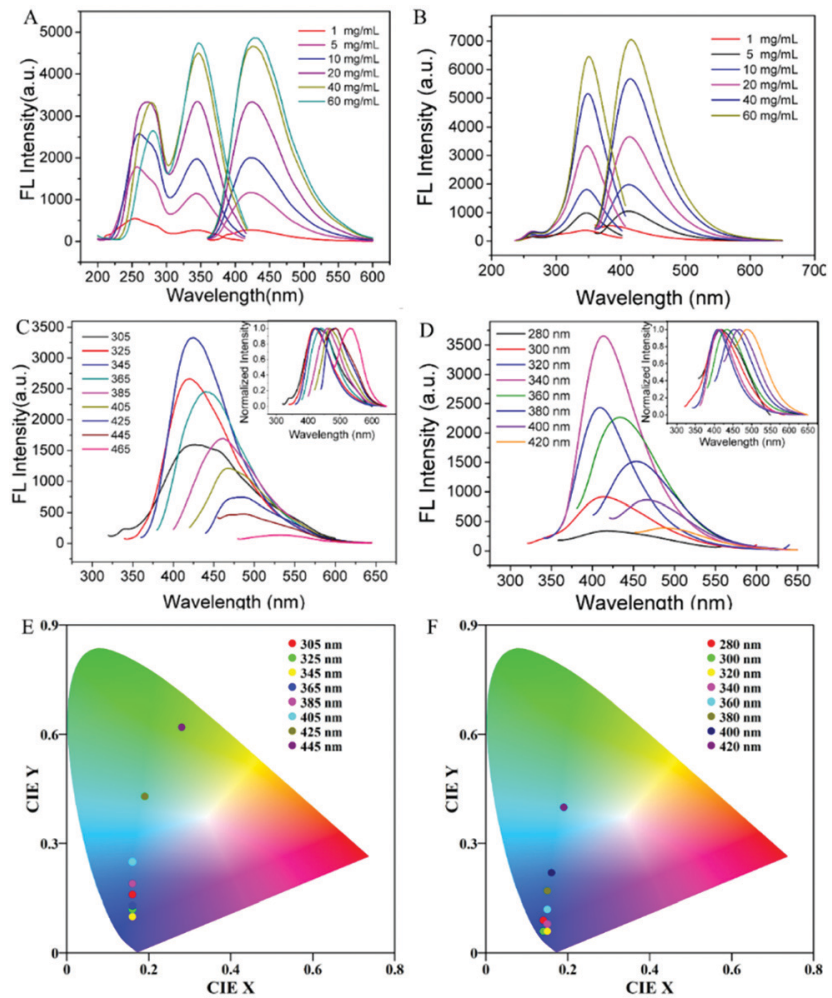

Fig. 4 (A) Emission spectra of P1 under different concentrations (mg mL $\mathrm{mL}^{-1}, \lambda_{\mathrm{ex}}=345 \mathrm{~nm}$ ); (B) emission spectra of P4 under different concentrations ( $\mathrm{mg} \mathrm{mL}^{-1}, \lambda_{\mathrm{ex}}=340 \mathrm{~nm}$ ); (C) excitation-dependent fluorescence of $\mathrm{P} 1$ at $20 \mathrm{mg} \mathrm{mL} \mathrm{mL}^{-1}$ (Inset: Normalized emission); (D) excitation-dependent fluorescence of P4 with at $20 \mathrm{mg} \mathrm{mL}^{-1}$ (Inset: Normalized emission); (E) CIE chromaticity coordinates obtained from the emission spectra of $\mathrm{P} 1$ at $20 \mathrm{mg} \mathrm{mL}^{-1}$; (F) CIE chromaticity coordinates obtained from the emission spectra from $\mathrm{P} 4$ at $20 \mathrm{mg} \mathrm{mL}^{-1}$.

fluorescence under UV light, while the fluorescence mainly originates from the electronic delocalization system.

Then we further studied the effect of excitation wavelength on PPA's fluorescence performance. As shown in Fig. 4C, the highest fluorescence intensity exists at the excitation wavelength of $345 \mathrm{~nm}$. When the wavelength is longer than $345 \mathrm{~nm}$, the fluorescence intensity decreases with the increase in wavelength, along with an obvious red-shift (Fig. 4C, inset). Afterward, Commission Internationale d'Éclairage (CIE) chromaticity coordinates of $\mathrm{P} 1$ solution at $20 \mathrm{mg} \mathrm{mL} \mathrm{mL}^{-1}$ were calculated from the emission spectra under various wavelengths and are shown in Fig. 4E. From Fig. 4E, we can see that the emission colours vary from blue to green, which is consistent with the clustering-tiggered emission mechanism. ${ }^{27-29}$ Compared with P1, the red-shift of P4 is not that obvious (Fig. 4F). Moreover, with the longer chain, P4 can only form local through-space conjugation, while P1 can form a through-space conjugation ring (confirmed by the subsequent DFT calculation results). Therefore, the absorption range of P4 narrows, resulting in a less obvious red-shift. ${ }^{30}$

Moreover, we obtained the fluorescence spectra, fluorescence lifetime and absolute quantum yield (QY) of pure PPA. The fluorescence spectra of pure P1 are shown in Fig. 5A. The
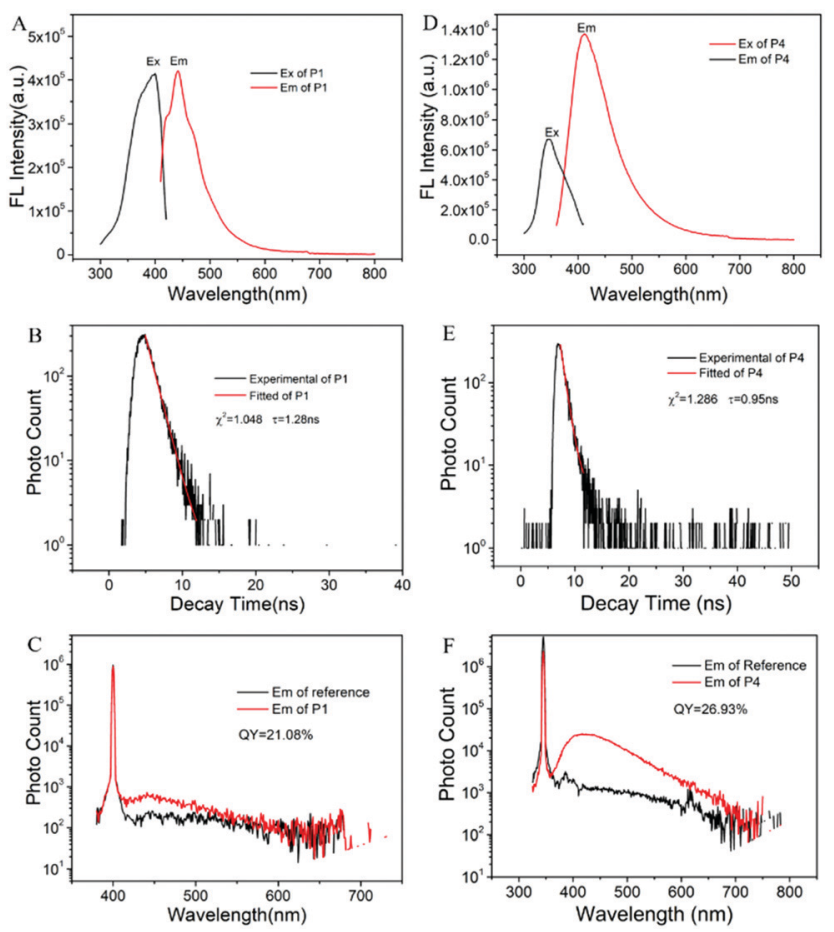

Fig. 5 (A) Excitation spectrum ( $E x, \lambda_{\mathrm{em}}=443 \mathrm{~nm}$ ) and emission spectrum (Em, $\lambda_{\mathrm{ex}}=399 \mathrm{~nm}$ ) of P1; (B) transient photoluminescence decay curve of P1 at $443 \mathrm{~nm}$ after excitation at $399 \mathrm{~nm}$; (C) absolute fluorescence quantum yield of $\mathrm{P} 1$ excited at $399 \mathrm{~nm}$; (D) excitation spectrum (Ex, $\lambda_{\mathrm{em}}=412 \mathrm{~nm}$ ) and emission spectrum (Em, $\lambda_{\mathrm{ex}}=345 \mathrm{~nm}$ ) of P4; (E) Transient photoluminescence decay curve of P4 at $412 \mathrm{~nm}$ after excitation at $345 \mathrm{~nm}$; (F) absolute fluorescence quantum yield of P4 excited at $345 \mathrm{~nm}$.

excitation band appears at $399 \mathrm{~nm}$, and the emission band appears at $443 \mathrm{~nm}$. Compared with the excitation and emission spectra of P1 solution in ethanol, the excitation and emission bands red-shift for about $50 \mathrm{~nm}$ and $20 \mathrm{~nm}$, respectively. This phenomenon is caused by the electron delocalization system formed in different chemical environments. Fig. 5B displays the fluorescence decay curve of P1 at $443 \mathrm{~nm}$ after the excitation at $399 \mathrm{~nm}$. The measured fluorescence lifetime of P1 is $1.28 \mathrm{~ns}$. Fig. $5 \mathrm{C}$ shows that the absolute QY of pure $\mathrm{P} 1$ is $21.08 \%$, which is relatively high among the non-aromatic AIE polymers. ${ }^{3,4}$

We also tested the fluorescence properties of $\mathrm{P} 4$ to systematically study the influence of chain length. By comparing Fig. 5A and D, we can see that the fluorescence intensity of P4 is stronger than that of P1. We believe that the longer chain in P4 increases the flexibility of the P4 molecules, resulting in the formation of denser luminogen clusters and leading to stronger fluorescence. The fluorescence lifetime of $\mathrm{P} 4$ is $0.95 \mathrm{~ns}$, which is shorter than that of P1, owing to the smaller energy gap (confirmed by subsequent DFT calculation). Besides, the QY of P4 is $26.93 \%$, slightly higher than that of P1 (Fig. 5C and F), which provides a possible method to improve the QY of non-aromatic AIE polymers.

Compared with other non-aromatic AIE polymers, especially poly(amidoamine)s, ${ }^{31-33} \mathrm{P}$ incorporated PPA shows outstanding fluorescence properties, including strong fluorescence 
intensity and high QY. ${ }^{21,34,35}$ The $\mathrm{P}$ incorporated PPA provides a possible method for the design of non-aromatic AIE polymers with high fluorescence intensity and QY.

\subsection{The fluorescence mechanism study}

According to the fluorescence properties of PPA, there is no doubt that the fluorescence intensity of PPA is related to its aggregate state. Hence, we explore the fluorescence mechanism of PPA through theoretical calculation and experiment investigation. Firstly, we employ density functional theory (DFT) at the level of B3LYP/6-31G (d) to obtain the optimized conformations and energy level of PPA (P1 and P4) with the Gaussian 09 program package. To simplify the calculation, we calculated the energy levels of the first-generation P1 and P4 with the molecule numbers increased from 1 to 4 (Table 1), and their optimized structures are displayed in Fig. S7 (ESI $\dagger$ ). With the increase in the molecule numbers of $\mathrm{P} 1$, the compact structure of $\mathrm{P} 1$ is formed due to the intermolecular $\mathrm{O} \cdots \mathrm{H}$ and $\mathrm{N} \cdots \mathrm{H}$ interactions $(1.949 \AA$, $2.059 \AA$, $2.050 \AA$, $2.113 \AA$, $2.035 \AA$, 2.149 , $2.202 \AA$ ) (Fig. 6A). The abundant hydrogen bonds diminish the distance between $\mathrm{P} 1$ molecules and rigidify the conformation of $\mathrm{P} 1$, which is beneficial to the formation of through-space conjugation. Similarly, intermolecular $\mathrm{O} \cdots \mathrm{H}$ and $\mathrm{N} \cdots \mathrm{H}$ interactions also promote the self-assembly of P4 (2.060 ̊, $2.217 \AA$, $2.134 \AA$ 足 $2.031 \AA, 2.270 \AA$ ) (Fig. 6C). These interactions could lead to the overlap of the electron clouds of $\mathrm{N}$ and $\mathrm{O}$, resulting in the formation of an electron delocalization system. At the same time, the HOMO-LUMO energy gap decreases as the number of P1/P4 molecules increases, which demonstrates that the molecules are more likely to be excited.

To further explore the fluorescence mechanism of PPA, we investigated the morphology of $\mathrm{P} 1$ at different concentrations

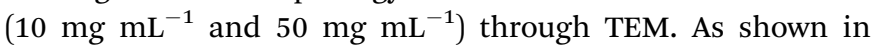
Fig. 7A, P1 assembles into loose irregular structures at a concentration of $10 \mathrm{mg} \mathrm{mL}^{-1}$. Nevertheless, at a concentration of $50 \mathrm{mg} \mathrm{mL}{ }^{-1}, \mathrm{P} 1$ assembles into spherical structures with uneven size (Fig. 7B and C). The compact conformation at high concentrations is conducive to forming clusters of $\mathrm{P} 1$, resulting in the stronger fluorescence intensity.

On the basis of the above theoretical calculations and investigation, we presume that the clusteroluminescence affected by the electron delocalization and molecular selfassembly is the origin of PPA's fluorescence. The fluorescence

Table 1 DFT calculation results of HOMO-LUMO energy levels of the conformations with 1-4 first-generation P1 and P4 molecules

\begin{tabular}{|c|c|c|c|c|c|}
\hline \multicolumn{2}{|c|}{$\begin{array}{l}\text { Molecules } \\
\text { number }\end{array}$} & \multirow{2}{*}{$\begin{array}{l}\text { E(HOMO) } \\
\text { (a.u.) } \\
-0.226\end{array}$} & \multirow{2}{*}{$\begin{array}{l}\begin{array}{l}\text { E(LUMO) } \\
\text { (a.u.) }\end{array} \\
0.066\end{array}$} & \multirow{2}{*}{$\begin{array}{l}\begin{array}{l}\text { Energy gap } \\
\text { (a.u.) }\end{array} \\
0.292\end{array}$} & \multirow{2}{*}{$\begin{array}{l}\begin{array}{l}\text { Energy gap } \\
(\mathrm{eV})\end{array} \\
7.946\end{array}$} \\
\hline $\mathrm{P} 1$ & 1 & & & & \\
\hline & 2 & -0.210 & 0.051 & 0.261 & 7.102 \\
\hline & 3 & -0.219 & 0.045 & 0.264 & 7.184 \\
\hline & 4 & -0.207 & 0.043 & 0.250 & 6.803 \\
\hline \multirow[t]{4}{*}{$\mathrm{P} 4$} & 1 & -0.226 & 0.065 & 0.291 & 7.919 \\
\hline & 2 & -0.208 & 0.062 & 0.270 & 7.347 \\
\hline & 3 & -0.205 & 0.047 & 0.252 & 6.857 \\
\hline & 4 & -0.204 & 0.045 & 0.249 & 6.776 \\
\hline
\end{tabular}

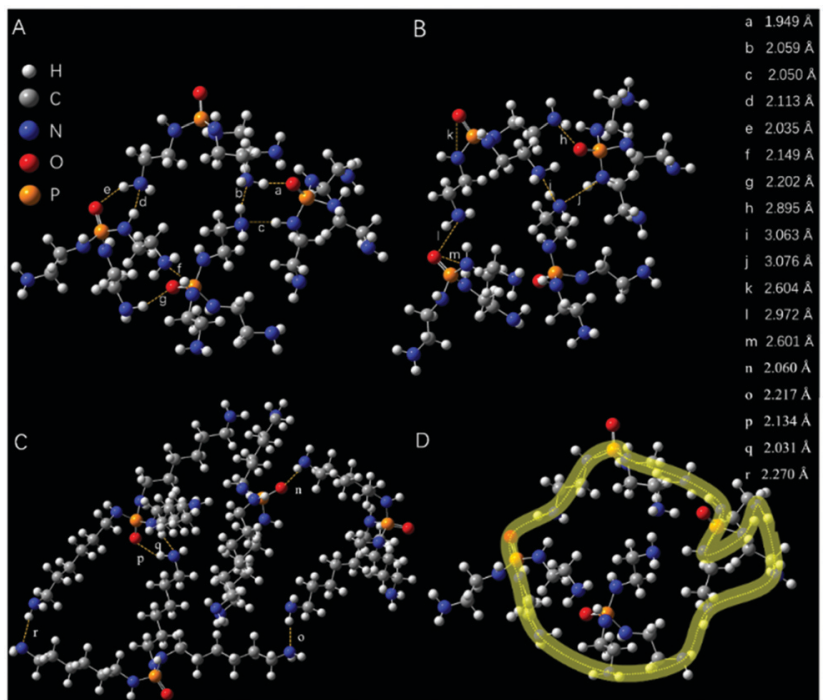

Fig. 6 (A) The accumulation of four P1 first-generation molecules induced by $\mathrm{H} \ldots \mathrm{O}$ and $\mathrm{H} \ldots \mathrm{N}$ strong intermolecular interactions; (B) intramolecular and intermolecular $\mathrm{O} \cdots \mathrm{N}$ and $\mathrm{N} \cdots \mathrm{N}$ interactions between four P1 molecules; (C) the accumulation of four P4 first-generation molecules induced by $\mathrm{H}$... O and $\mathrm{H} \cdots \mathrm{N}$ strong intermolecular interactions; (D) schematic diagram of the through-space conjugation for P1.

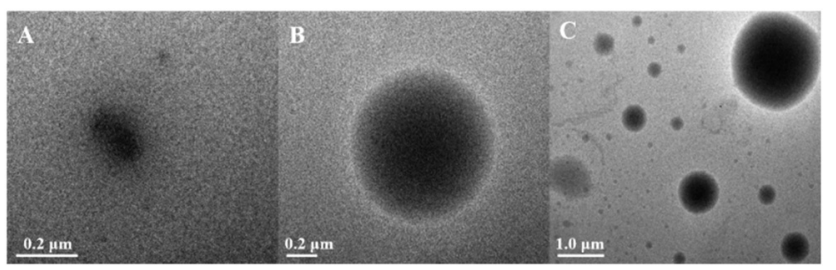

Fig. 7 Micrograph of P1 self-assembly morphology in ethanol with concentrations of $10 \mathrm{mg} \mathrm{mL}^{-1}(\mathrm{~A})$ and $50 \mathrm{mg} \mathrm{mL}^{-1}(\mathrm{~B}-\mathrm{C})$.

mechanism schematic diagram of PPA is shown in Fig. 8. As the concentration of PPA or ratio of poor solvent increases, the PPA tends to form a tighter cluster owing to the intermolecular hydrogen bonds, leading to stronger fluorescence-emission.

\section{6 pH dependency}

Considering that PPA contains a large amount of $\mathrm{O}$ and $\mathrm{N}$ atoms, we further explored the $\mathrm{pH}$ dependency property of P1. The $\mathrm{pH}$ responsiveness of $\mathrm{P} 1$ was investigated at different

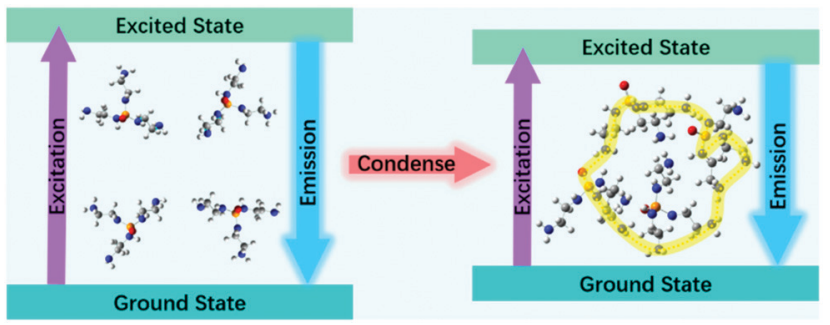

Fig. 8 Fluorescence mechanism schematic diagram of PPA. 
$\mathrm{pH}$ ranging from 2 to 12 with a $\mathrm{P} 1$ concentration of $5 \mathrm{mg} \mathrm{mL}{ }^{-1}$. The original $\mathrm{pH}$ of the $\mathrm{P} 1$ aqueous solution is 8.48 (Fig. S8, $\mathrm{ESI} \dagger$ ), and the fluorescence intensity is strongest when $\mathrm{pH}=4$. When the $\mathrm{pH}$ value is in the range of $4-12$, as the $\mathrm{pH}$ value increases, the intramolecular and intermolecular hydrogen bonds between P1 molecules are affected by increasing $\mathrm{OH}^{-}$, and this will lead to the loose structure of P1 with weak fluorescence. When the $\mathrm{pH}$ value is below 4 , the amino groups in P1 will combine with the hydrogen ions to form ammonium ions, resulting in a repulsive force between $\mathrm{P} 1$ molecules. With the formation of the looser conformation of P1, a decrease in the fluorescence intensity of P1 is observed. Due to the $\mathrm{pH}$ dependency property, PPA is expected to be used as a $\mathrm{pH}$ probe in the chemical sensing field.

\subsection{Ion probe}

To our surprise, the addition of PPA can change the colour of copper chloride solution from colourless to purple without any effect on the fluorescence of PPA (Fig. 9A and Fig. S9, ESI $\dagger$ ). This is attributed to the large amount of phosphoryl bonds contained in the synthesized PPA. After adding PPA to copper chloride solution, the secondary amines in the phosphoryl bonds are complexed with the copper ions. While the coordination complex shows a purple colour, thereby making the solution turn from colourless to purple (Scheme S2, ESI $\dagger$ ). The secondary amine groups have no effect on the fluorescence of PPA, so its fluorescence intensity does not change. Then we analyzed the relationship between the absorbance of coordination complex solution (with a P1 concentration of $20 \mathrm{mg} \mathrm{mL} \mathrm{m}^{-1}$ ) and the concentration of copper ions (Fig. 9B). It is obvious that the absorbance increased linearly with the increase in the concentration of copper ions $\left(R^{2}=0.9996\right)$. The detection limit is $0.15 \mathrm{mmol} \mathrm{L}{ }^{-1}$ in theory. Besides, the other metal ions $\left(\mathrm{Na}^{+}\right.$, $\mathrm{Cd}^{2+}, \mathrm{Fe}^{3+}, \mathrm{Hg}^{2+}, \mathrm{Co}^{2+}, \mathrm{Al}^{3+}, \mathrm{Zn}^{2+}$ ) have no such effect, for they cannot complex with PPA and form coloured coordination complexers (Fig. S10, ESI $\dagger$ ). We also found out that the solution colour will change to wathet blue (Fig. S11, ESI $\dagger$ ) after removing $\mathrm{Cu}^{2+}$ by ethylenediaminetetraacetic acid disodium salt (EDTA$2 \mathrm{Na}$ ). Furthermore, we explored the biocompatibility of PPA by CCK-8 assays, ${ }^{36}$ and the results are shown in Fig. S12 (ESI $\dagger$ ). We can see that the cell viability is more than $84 \%$ after incubation for $24 \mathrm{~h}$ with a concentration of PPA as high as $1.0 \mathrm{mg} \mathrm{mL} \mathrm{mL}^{-1}$, indicating the excellent biocompatibility of PPA. This discovery
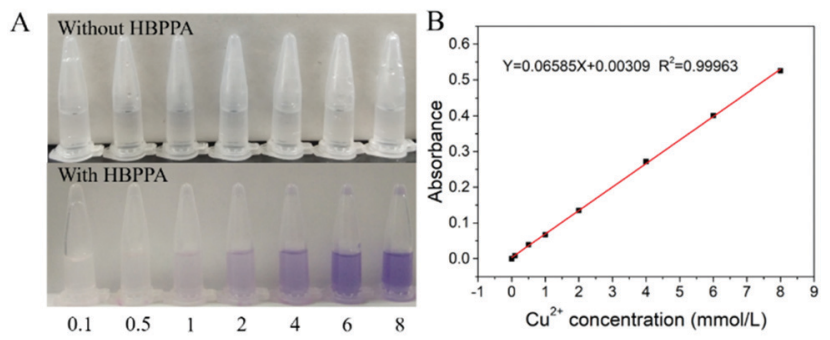

Fig. 9 (A) The colour change phenomenon of PPA aqueous solution (20 $\mathrm{mg} \mathrm{mL}^{-1}$ ) with different concentrations of $\mathrm{Cu}^{2+}\left(\mathrm{mmol} \mathrm{L}^{-1}\right)$. (B) The relationship between absorbance and the concentration of $\mathrm{Cu}^{2+}$. provides an effective and environmentally friendly means for the qualitative and quantitative detection of copper ions.

\section{Conclusions}

In summary, a green and convenient method is proposed for the synthesis of PPA through an ester ammonolysis reaction by TEP and EDM or DAH instead of polycondensation by phosphorus oxychloride and diamine. The by-products are facile to separate and harmless to the environment. What is more, PPA could emit strong blue fluorescence under UV light and shows an obvious AIE character. This is attributed to the fact that PPA could assemble into aggregates and form a through-space conjunction due to intermolecular and intramolecular hydrogen bonds and the interactions between phosphoryl bonds and amino groups. Such aggregation is helpful to the formation of luminogen clusters, resulting in the fluorescence of PPA. The molecular weight, amino groups and chain length are all important factors that effectively affect the PPA's fluorescence intensity, HOMO-LUMO energy gap and quantum yield. What is more, PPA is sensitive to $\mathrm{Cu}^{2+}$ and can change the colour of copper chloride solution from colourless to purple, realizing the qualitative and quantitative visual detection of $\mathrm{Cu}^{2+}$ instead of time-consuming and expensive $\mathrm{Cu}^{2+}$ detection methods. This work provides an innovative method for PPA synthesis, as well as an enrichment of $\mathrm{P}$ incorporated AIE polymers and an in situ visual detection agent for $\mathrm{Cu}^{2+}$.

\section{Author contributions}

L. G.: synthesis, structural analysis, writing - original draft. H. Y.: supervision of conceptualization and methodology. L. W.: synthesis, data plotting, writing - original draft. P. Y.: DFT calculation, structural analysis. L. Y.: TEM experiment. Y. Z.: writing - polishing.

\section{Conflicts of interest}

There are no conflicts to declare.

\section{Acknowledgements}

This work was supported by the National Natural Science Foundation of China [21875188; 22175143]; Open Fund of Guangdong Provincial Key Laboratory of Luminescence from Molecular Aggregates, Guangzhou 510640, China (South China University of Technology) [2019B030301003] and Innovation Foundation for Doctor Dissertation of Northwestern Polytechnical University (CX2021108).

\section{Notes and references}

1 J. Mei, N. Leung, R. Kwok, J. Lam and B. Z. Tang, Chem. Rev., 2015, 115, 11718-11940. 
2 J. Yang, Z. Chi, W. Zhu, B. Z. Tang and Z. Li, Sci. China: Chem., 2019, 62, 1090-1098.

3 W. Yuan and Y. Zhang, J. Polym. Sci., Part A: Polym. Chem., 2017, 55, 560-574.

4 D. Tomalia, B. Klajnert-Maculewicz, K. Johnson, H. Brinkman, A. Janaszewska and D. Hedstrand, Prog. Polym. Sci., 2019, 90, 35-117.

5 Y. Feng, H. Yan, F. Ding, T. Bai, Y. Nie, Y. Zhao, W. Feng and B. Z. Tang, Mater. Chem. Front., 2020, 4, 1375-1382.

6 D. Wu, Y. Liu, C. He and S. Goh, Macromolecules, 2005, 38, 9906-9909.

7 L. Yuan, H. Yan, L. Bai, T. Bai, Y. Zhao, L. Wang and Y. Feng, Macromol. Rapid Commun., 2019, 40, 1800658.

8 L. Bai, H. Yan, L. Wang, T. Bai, L. Yuan, Y. Zhao and W. Feng, Macromol. Mater. Eng., 2020, 305, 2000126.

9 R. Wang, W. Yuan and X. Zhu, Chin. J. Polym. Sci., 2015, 33, 680-687.

10 R. Restani, P. Morgado, M. Ribeiro, I. Correia, A. AguiarRicardo and V. Bonifácio, Angew. Chem., Int. Ed., 2012, 51, 5162-5165.

11 S. Niu, H. Yan, S. Li, P. Xu, X. Zhi and T. Li, Macromol. Chem. Phys., 2016, 217, 1185-1190.

12 Y. Feng, T. Bai, H. Yan, F. Ding, L. Bai and W. Feng, Macromolecules, 2019, 52, 3075-3082.

13 L. Bai, H. Yan, T. Bai, Y. Feng, Y. Zhao, Y. Ji, W. Feng, T. Lu and Y. Nie, Biomacromolecules, 2019, 20, 4230-4240.

14 S. Niu, H. Yan, Z. Chen, L. Yuan, T. Liu and C. Liu, Macromol. Rapid Commun., 2016, 37, 136-142.

15 F. Liao, Y. Ju, X. Dai, Y. Cao, J. Li and X. Wang, Polym. Degrad. Stab., 2015, 120, 251-261.

16 X. Hu, Y. Guo, L. Chen, X. Wang, L. Li and Y. Wang, Polym. Degrad. Stab., 2012, 97, 1772-1778.

17 Q. Lv, J. Huang, M. Chen, J. Zhao, Y. Tan, L. Chen and Y. Wang, Ind. Eng. Chem. Res., 2013, 52, 9397-9404.

18 F. Liao, L. Zhou, Y. Ju, Y. Yang and X. Wang, Ind. Eng. Chem. Res., 2014, 53, 10015-10023.
19 W. Xu, Y. Wang, S. Li, Z. Ke, Y. Yan, S. Li, Z. Xing, C. Wang, F. Zeng, R. Liu and F. Deng, RSC Adv., 2015, 5, 5425-5432.

20 J. Köhling, V. Kozel, V. Jovanov, R. Pajkert, S. Tverdomed, O. Gridenco, M. Fugel, S. Grabowsky, G. Röschenthaler and

V. Wagner, ChemPhysChem, 2019, 20, 665-671.

21 T. Baumgartner, Acc. Chem. Res., 2014, 47, 1613-1622.

22 S. Padghan, R. Bhosale, S. Bhosale, F. Antolasic, M. Kobaisi and S. Bhosale, Molecules, 2017, 22, 1417.

23 J. Zhang, S. Devaramani, D. Shan and X. Lu, Anal. Bioanal. Chem., 2016, 408, 7155-7163.

24 C. Karadaş and D. Kara, Food Chem., 2017, 232, 242-248.

25 I. Rapp, C. Schlosser, D. Rusiecka, M. Gledhill and E. Achterberg, Anal. Chim. Acta, 2017, 976, 1-13.

26 V. Bekiari, P. Lianos, U. Stangar, B. Orel and P. Judeinstein, Chem. Mater., 2000, 12, 3095-3099.

27 Q. Zhou, Z. Wang, X. Dou, Y. Wang, S. Liu, Y. Zhang and W. Yuan, Mater. Chem. Front., 2019, 3, 257-264.

28 X. Dou, Q. Zhou, X. Chen, Y. Tan, X. He, P. Lu, K. Sui, B. Tang, Y. Zhang and W. Yuan, Biomacromolecules, 2018, 19, 2014-2022.

29 S. Zheng, T. Zhu, Y. Wang, T. Yang and W. Yuan, Angew. Chem., Int. Ed., 2020, 59, 1-6.

30 Y. Feng, H. Yan, F. Ding, T. Bai, Y. Nie, Y. Zhao, W. Feng and B. Tang, Mater. Chem. Front., 2020, 4, 1375-1382.

31 S. Lin, T. Wu, Y. Jao, C. Liu, H. Lin, L. Lo and C. Yang, Chem. - Eur. J., 2011, 17, 7158-7161.

32 S. Koley and S. Ghosh, Phys. Chem. Chem. Phys., 2016, 18, 24830-24834.

33 W. Yang, X. Wu, F. Liu, Y. Dou, Z. Hu and W. Hao, RSC Adv., 2016, 41, 34252-34260.

34 I. Manners, J. Polym. Sci., Part A: Polym. Chem., 2002, 40, 179-191.

35 T. Baumgartner and R. Réau, Chem. Rev., 2006, 106, 4681-4727.

36 C. Yan, N. Zhang, P. Guan, P. Chen, S. Ding, T. Hou, X. Hu, J. Wang and C. Wang, Int. J. Biol. Macromol., 2020, 153, 723-735. 